\title{
Life science students' attitudes, interest, and performance in introductory physics for life sciences: An exploratory study
}

\author{
Catherine H. Crouch, ${ }^{1}$ Panchompoo Wisittanawat, ${ }^{1,2}$ Ming Cai, ${ }^{2}$ and K. Ann Renninger ${ }^{2}$ \\ ${ }^{1}$ Department of Physics \& Astronomy, Swarthmore College, Swarthmore, Pennsylvania 19081, USA \\ ${ }^{2}$ Department of Educational Studies, Swarthmore College, Swarthmore, Pennsylvania 19081, USA
}

(Received 26 July 2016; revised manuscript received 28 July 2017; published 5 March 2018)

\begin{abstract}
In response to national calls for improved physical sciences education for students pursuing careers in the life sciences and medicine, reformed introductory physics for life sciences (IPLS) courses are being developed. This exploratory study is among the first to assess the effect of an IPLS course on students' attitudes, interest, and performance. The IPLS course studied was the second semester of introductory physics, following a standard first semester course, allowing the outcomes of the same students in a standard course and in an IPLS course to be compared. In the IPLS course, each physics topic was introduced and elaborated in the context of a life science example, and developing students' skills in applying physics to life science situations was an explicitly stated course goal. Items from the Colorado Learning about Science Survey were used to assess change in students' attitudes toward and their interest in physics. Whereas the same students' attitudes declined during the standard first semester course, we found that students' attitudes toward physics hold steady or improve in the IPLS course. In particular, students with low initial interest in physics displayed greater increases in both attitudes and interest during the IPLS course than in the preceding standard course. We also find that in the IPLS course, students' interest in the life science examples is a better predictor of their performance than their pre-IPLS interest in physics. Our work suggests that the life science examples in the IPLS course can support the development of student interest in physics and positively influence their performance.
\end{abstract}

DOI: 10.1103/PhysRevPhysEducRes.14.010111

\section{INTRODUCTION}

As the understanding of the physical basis of biology increases, and as physics-based technological tools are integrated throughout both biological research and clinical medicine, physics is becoming increasingly valuable in the life scientist's toolkit [1-3]. Multiple efforts are underway to improve introductory physics for students in the life sciences and allied health fields [4-6]. A common strategy for reforming such courses includes designing the course to help students make connections between physics and the life sciences, modifying the syllabus to focus on topics and skills most important for biology, and giving the students numerous opportunities to apply physics to "authentic" [7] biological examples—examples in which a biology student or biologist would agree that physics provides useful insights into a biological situation. Courses designed in this way are often referred to as introductory physics for life sciences (IPLS) courses.

Published by the American Physical Society under the terms of the Creative Commons Attribution 4.0 International license. Further distribution of this work must maintain attribution to the author(s) and the published article's title, journal citation, and DOI.
In our IPLS course, life science examples, such as the optics of vision and the electrical basis of nerve signaling, are central to the course content, and many are revisited multiple times, both qualitatively and quantitatively, and in different settings (i.e., in class, in lab, and on the homework). This provides frequent opportunities for students to work with, reflect on, and make meaningful connections between the life sciences and the physics they need to learn. Such connections are intended to enable students to identify the utility of learning physics [8], positively influence their feelings about physics, and hopefully support the development of their interest in physics [9-12].

In the present study, life science student participants took a standard first semester of introductory physics followed by the second semester IPLS course. The first semester course provides comparison data for studying the same students' attitudes toward, interest in, and performance during the IPLS course.

We address two research questions: (i) What effect does the IPLS context, and the use of life science examples more specifically, have on students' attitudes and beliefs about physics and learning physics (henceforth, "attitudes"), interest, and performance in the IPLS course? (ii) What is the relationship between interest in physics, interest in the life science examples of the IPLS course, and performance? 
To address these questions, student attitudes toward physics were measured at the beginning and the end of each semester using the Colorado Learning About Science Survey (CLASS) [13]. In addition, we measure two different types of interest: interest in physics, and interest in the life science examples. To measure students' interest in physics, we used items from the CLASS that map onto indicators of interest conceptualized as a developmental variable $[10,12]$. To assess students' interest in the life science examples, we designed an end-of-semester survey. Finally, to measure student performance, we used composite exam scores.

Our core findings are twofold. First, in the standard course, all students' attitudes toward physics declined, and their interest in physics did not change. During the second semester IPLS course, all students' attitudes held steady or improved. Moreover, students who entered the IPLS course with low interest in physics ("low initial interest" students) made significant gains in both overall attitudes and interest (effect size $=0.5$ or 0.6 depending on measure, statistics presented in Sec. V). Students with the highest initial interest in physics showed a decline in interest; however, this decrease may be either a ceiling effect or the effect of very high expectations, as this group of students entered the IPLS course with an extremely high average initial physics interest ( $93 \%$ favorable on the selected items), as discussed in Sec. V. B.

A second core finding is that in this IPLS course, students' interest in the life science examples explains more of the variance in students' exam scores than does their interest in physics when entering the IPLS course. As in general, the literature on interest shows that interest supports performance, this finding suggests that engaging students' interest through integral life science examples may be particularly beneficial for students who begin a required physics course with low initial interest in physics.

Our results suggest that IPLS courses that leverage students' interest in the life sciences through the strategies used here hold promise for engaging and supporting the development of life science students' interest in physics. Given that this work is the first reported study of interest and its role in student engagement and learning in the IPLS course, as well as one of the first studies of attitudes in IPLS courses, our findings not only document the encouraging outcomes of our particular IPLS course, but also suggest that student interest and its development are fruitful directions for further investigation by the IPLS community.

\section{ATTITUDES TOWARDS AND INTEREST IN PHYSICS}

\section{A. Previous use of the CLASS to study attitudes and interest}

The CLASS [13] is a validated survey that assesses student attitudes towards and beliefs about learning physics by comparing students' self-reported attitudes to those of experts (professional physicists). The 42 CLASS items probe matters ranging from students' expectations about the amount and type of effort required to learn physics to their own enjoyment of physics and expectations for success; students indicate their level of agreement with the statements on a 5-point Likert scale. Its items were designed to elucidate student perceptions of physics, attitudes towards physics, and expectations about learning physics, based on previous research [14-17], as well as to inform common instructor concerns [18].

Items from the CLASS have previously been used to measure interest in two different ways. Developers of the CLASS [13] assigned the items to eight categories, including a "Personal Interest" category, using reduced basis factor analysis. In a subsequent validation study of the CLASS $(N=3844)$, Douglas et al. [19] identified a Personal Application Factor that included a slightly different set of items. Neither of these studies focused on interest, nor did they study interest as a variable that develops. In the present study, we developed a theoretically based metric, selecting those CLASS items that reflect the understanding of interest from motivation research, and formed a factor described further in Sec. IV. D.

Extensive work, including a 24-study meta-analysis of studies about student attitudes toward learning physics [20], indicates that more expertlike attitudes do not generally develop unless a course specifically cultivates them. In fact, attitudes have been found to decline in algebrabased and calculus-based courses that do not explicitly cultivate expertlike attitudes or teach modeling skills, even if these courses are taught with reformed pedagogies that lead to substantial learning gains [20]. Studies of second semester courses are less consistently negative; one 41-student study reported substantial declines in average CLASS responses during the first semester of calculusbased physics for engineers and physics majors, followed by modest improvements by the same students during the second semester [13]. On the other hand, declines in CLASS responses were identified during a four-year study of both the first and second semester courses of a largeenrollment calculus-based second semester course, although the second semester declines are smaller [21]. Similarly, at the University of Minnesota, CLASS responses were also found to decline during both semesters of an algebra-based course for life science students, even though the courses employed Peer Instruction in lecture and cooperative group problem solving in discussion sections [22]. Studies of the University of Minnesota IPLS courses (taught with the same pedagogy), however, suggested that the IPLS approach can have positive effects on attitudes. CLASS improvements were found in both IPLS semesters [22]. Little work has been undertaken to investigate the relationship between attitudes and student learning, and, like results from the study of attitude and pedagogy, findings specific to learning are mixed [23,24]. 


\section{B. The developmental model of interest}

Whereas the word "interest" in everyday discussion is typically used to refer to positive feelings, or to how much someone likes something, research defines interest to include both a person's psychological state during engagement with content (e.g., physics) and a predisposition to engage repeatedly with that content over time $[10,12]$. Affective neuroscience has now established that all humans are hardwired to engage in seeking behaviors that may provide more information about particular content, suggesting that learners can be supported to develop their interest for physics, and can develop new interests [12]. The development of interest leads to wanting to continue to engage in knowledge seeking and learning, and is characterized by changes in the relation among levels of knowledge and the corresponding values and feelings. Importantly for pedagogy, interest has the potential to be supported to develop at any age and in any context; phases in the development of interest have been identified as initiating with the triggering of momentary attention to particular content that may or may not be sustained, and extending through the development of well-developed interest $[10,12]$.

Conceptualized in this way, interest differs from the study of students' attitudes toward physics and physics learning by the CLASS. Although liking can be a proxy for attitude, it is not a sufficient indicator of interest, because it does not distinguish between phases in interest development [12]. It is possible for persons who are new to developing an interest to "like" some content, and for those who have a more developed interest to also like it. Moreover, it is possible for liking to be distinct from "wanting to learn;" learners who like physics, for example, do not necessarily also want to learn physics [25]. ${ }^{1}$

Not surprisingly, perhaps, study of interest as a variable that develops has identified differences between learners with less developed interest in a discipline such as physics, whose interest may only be fleeting (e.g., the enthusiastic reaction most students have to a showy demonstration in class), and learners whose interest is more sustained, who will invest effort in order to seek knowledge of the discipline [26]. Wanting to learn indicates the learner has moved beyond the earliest stage of interest development. Differences in the phase of interest have implications for pedagogy. More developed interest facilitates the development of attention, goal setting, and learning strategies [10,11]. It also enables individuals to expend effort without it feeling effortful; in the case of learning science, this enables the learner to persist through the frustration of a

\footnotetext{
${ }^{1}$ The Douglas et al. analysis of the CLASS [19] initially found that item 25 , "I enjoy solving physics problems," did not factor, but they used modification indices to retain it as part of their factor, because of their research team's judgment of the importance of this item to their conception of interest as an attitude.
}

difficult problem set or an experiment gone awry [27]. In contrast, learners with less developed interest may need support to make connections to the content to be learned (e.g., examples from their everyday experience can help them to make this type of connection); such support can promote attention to the content, and use learning goals and strategies that enable them to engage in deeper learning of the content $[11,12,28,29]$.

In short, supporting the development of interest has been found to facilitate the kind of productive learning behaviors that lead to successful science learning [12,30]. Moreover, a student is more likely to persevere in the face of challenge if he or she has been taught in a manner that deliberately supports the development of interest.

Strategies found to be successful in promoting the development of interest include (though are not limited to) the following:

(i) using content that is of interest to students as the context for new ideas $[9,31,32]$, and

(ii) enabling recognition of utility [8] or meaningfulness [33] of the content to be learned.

Our IPLS course, by being organized around authentic life science examples, seeks to support the development of students' interest through these strategies.

Interest has been shown to serve the same function for both male and female students' learning (e.g., it facilitates meaningful engagement), although the specific topics that are of interest can vary with gender. For example, in one set of studies, Hoffmann and Häussler found that female students learned more when the study of pumps focused on cases that were of interest to females (e.g. heart pumps) rather than those that were not (oil pumps), whereas male students, with equivalent interest in both, learned similarly in both contexts [9]. Randler and Bogner, on the other hand, reported that when male and female students had comparable interest in a topic (e.g., ecology), there were no differences in their achievement [34]. Other work suggests that once interest in a topic has developed, few differences among female and male students exist [35]. This suggests that the specific choice of topics in an IPLS course may be less important than the more general presence of life science contexts as contexts for learning physics.

\section{INTRODUCTORY PHYSICS FOR LIFE SCIENCES (IPLS) AT SWARTHMORE}

At Swarthmore College, life science students take a standard first semester of calculus-based introductory physics, alongside engineering students and chemistry majors. This is followed by a second semester IPLS course covering optics, electricity, magnetism, and waves [22]. (Prior to Fall 2015, only the second semester was revised due to staffing limitations.) Nearly all students who enroll in this second semester course are biochemistry or biology majors, and/or pre-medical students, along with a few engineers interested in biomedical engineering. 
In this course, developed by one of us (C. H. C.), ${ }^{2}$ each physics topic is introduced using a biological example to provide context, drawing on the expansive framing approach of Engle et al. [36,37] in order to support interest development and learning. Expansive framing is an approach to curriculum design in which learners (in this case, life science students) are supported to understand that their prior understanding is relevant to new learning (in this case, physics), and encouraged to draw on prior knowledge in the course of new learning. Subsequently, these biological examples are explored further through in-class conceptual questions and examples, laboratory activities, and homework problems. Consequently, the students encounter each of the contexts at least twice, and often 3-5 times.

For example, a central idea in cell biology is the existence of an electric potential difference across the cell membrane ("membrane potential"), produced by differing concentrations of ions on either side. The arrangement of charge on the cell membrane is used to introduce the study of electric fields of extended arrangements of charge, and then as a case of a pair of parallel sheets of charge with uniform density and opposite charge. Subsequently, the membrane potential provides the primary illustration for studying electric potential energy, potential difference, and potential; it is used in several conceptual questions in class and homework problems after class.

The membrane potential is also used to demonstrate storing electrical energy and releasing it to accomplish some purpose. Capacitors are introduced as a general type of which the cell membrane is an example, and students do homework problems about cell membrane capacitance, energy storage, and the effect of the dielectric constant of the membrane material. Finally, in the discussion of batteries, the electrochemical equilibrium across the membrane (maintained by a combination of differential permeabilities and active pumping of ions) is given as an illustration of a battery, and students do problems about the energetics of moving ions across the cell membrane by analogy to circuits as well as from ideas of electric potential and potential energy.

\footnotetext{
${ }^{2}$ To develop the course, C.H.C. (a chemical physicist with some background and expertise in biophysics, as well as several family members who are physicians) prepared an initial syllabus and list of life science examples, based on the BIO 2010 report and her knowledge of applications of physics in the life sciences and medicine. She then met with biology and biochemistry colleagues to get their input on the proposed syllabus, and to identify examples of how the physics topics appeared in their courses or in their research topics and techniques. She also consulted with other physicists undertaking similar course development and examined existing resources for introductory and intermediate courses in physics for biology and medicine. Finally, she used her expertise in research-validated physics pedagogies and curricular materials to adapt existing pedagogies and materials for use in the new course.
}

Throughout the study of each topic, students also engage with questions, problems, and labs that do not involve any biological connection. For example, in the study of capacitors, students also consider the uses of capacitors in defibrillators, camera flashes, and the NOVA fusion experiment, and go through a sequence of qualitative reasoning about capacitance and capacitors inspired by that used in the Tutorials in Introductory Physics [38]. The biological examples serve to frame the course and provide context, but each topic involves nonbiological problems as well.

The class of roughly 40 students meets three hours per week, and also includes a weekly three-hour laboratory taught in sections of up to 15 students. Peer Instruction [39] is used during the large class meetings; several of the laboratories are based on the Tutorials in Introductory Physics [38] or are problem based [40-42]. Students can also attend optional problem solving meetings twice a week, where they can work on the assigned homework problems with other students or receive help from student course assistants. Table I provides an abbreviated syllabus for the IPLS course including the biological examples. A more detailed syllabus and additional course materials are available as Supplemental Material [43].

\section{METHODS}

\section{A. Study design}

We examine changes in students' attitudes towards physics, as measured by their pre- and post-semester responses to the CLASS, and interest in physics, using a subset of the CLASS items, and compare the same students' responses obtained in a standard first-semester course in introductory physics to those obtained in a second semester IPLS course. Using an end-of-semester survey, we also examine students' self-reported interest in the life science examples used in the IPLS course. Finally, we examine IPLS course performance, using students' combined exam $z$ scores from the second semester IPLS course, and its relationship with students' interest in physics and in the life science examples. Registration records and an enrollment form (completed after the initial class meeting of the semester) provide information about gender, mathematics and physics background, and students' goals for taking the course.

Data were obtained from three consecutive offerings of the second semester IPLS course. Analyses identified few year-by-year differences, indicating the appropriateness of aggregating all years of data for subsequent analysis. The IPLS course was taught by the same instructor (C. H. C.) all three years of the study. Because of staffing needs, the standard course was taught by two other instructors (one in Year 1, one in Years 2 and 3). Based on positive student course evaluations, we know both are well-liked faculty who are highly dedicated and perceived as supportive of students. All instructors used Peer Instruction. 
TABLE I. List of physics topics with biological contexts. Boldface examples, the most frequently used and most prominent, were the basis of the end-of-semester survey used to determine example interest.

\begin{tabular}{ll}
\hline \hline Physics topic & \multicolumn{1}{c}{ Biological contexts } \\
\hline Geometric optics & Pinhole vision in invertebrates \\
& Human vision and vision correction \\
& Microscope optics \\
Electrostatics (electric force, field, & Electrostatics in molecular interactions \\
potential energy, potential differ-ence, & Cell membrane potential \\
potential) & Electrocardiography \\
& Faraday cage shielding for neurophysiology measurements \\
& Debye shielding of electrical interactions in salt water \\
Circuits (consisting of resistors, batteries, & Dielectric constant of water reducing chemical binding energies \\
and capacitors) & Gel electrophoresis \\
Magnetic interactions & Nerve signaling (passive spread, action potential propagation) \\
& Magnetic navigation (magnetotactic bacteria) \\
Electromagnetic induction & NMR spectroscopy \\
& Pacemaker safety \\
Interference and diffraction & Chemical shifts in NMR \\
& X-ray determination of molecular structure \\
& Structural color in insects \\
\hline \hline
\end{tabular}

\section{B. Participants}

Participating students were enrolled in one of three offerings of a second semester IPLS course. Only students who took both the standard first semester course and the IPLS second semester course and completed all surveys were included in the study; their data were aggregated for analysis. Table II provides descriptive information about the participants based on the students' registration records and their course enrollment form. There were no substantial differences between the three offerings and the enrollments in each section were comparable all three years. (In Year 1, the enrollment was roughly twice that of Years 2 and 3, because the course had only been offered every other year up to that point; that year the course was divided into two sections. The substantial enrollment led to resources being allocated to offer it yearly thereafter.)

\section{Data sources}

Data sources for this study included registration records that provide students' class year, major or intended major, and gender; a course enrollment form; students' pre- and postresponses to the CLASS [13] and the end-of-semester survey; and students' composite exam scores.

On the enrollment form, students were asked to write a brief summary of their reasons for taking the course. Based on these open-ended responses, students' goals were

TABLE II. Characteristics of students who completed all surveys, aggregated over the three years of the study. The total number of enrolled life science students over three years was 156; the group studied was representative of the total enrollment in all of these characteristics, as verified by independent $t$ tests. Students with "minimal" math background had only completed the prerequisite of a semester of single-variable calculus; "medium" had completed the second semester of single-variable calculus; and "high" had completed further mathematics (linear algebra and/or multivariable calculus).

\begin{tabular}{|c|c|c|c|c|c|c|}
\hline \multicolumn{7}{|l|}{$N_{\text {total }}=83$} \\
\hline Major & Biology & Biochemistry & $\begin{array}{l}\text { Neuroscience } \\
\text { or psychology }\end{array}$ & $\begin{array}{l}\text { Premedical, } \\
\text { nonscience major }\end{array}$ & $\begin{array}{l}\text { Physical } \\
\text { science major }\end{array}$ & Other \\
\hline & $33(39.8 \%)$ & $8(9.6 \%)$ & $16(19.3 \%)$ & $8(9.6 \%)$ & $11(13.3 \%)$ & $7(8.4 \%)$ \\
\hline Gender & $\begin{array}{c}\text { Male } \\
38(45.8 \%)\end{array}$ & $\begin{array}{c}\text { Female } \\
45(54.2 \%)\end{array}$ & & & & \\
\hline Goals & $\begin{array}{c}\text { Requirement } \\
11(13.3 \%)\end{array}$ & $\begin{array}{c}\text { Learning } \\
27(32.5 \%)\end{array}$ & $\begin{aligned} & \text { Both } \\
38 & (45.8 \%)\end{aligned}$ & $\begin{array}{c}\text { Unreported } \\
7(8.4 \%)\end{array}$ & & \\
\hline Math preparation & $\begin{array}{c}\text { Minimal } \\
24(28.9 \%)\end{array}$ & $\begin{array}{c}\text { Moderate } \\
25(30.1 \%)\end{array}$ & $\begin{array}{c}\text { High } \\
30(36.1 \%)\end{array}$ & $\begin{array}{c}\text { Unreported } \\
4(4.8 \%)\end{array}$ & & \\
\hline
\end{tabular}


TABLE III. Interest metrics compared in the study, listed by CLASS item number. The Personal Interest Category was designed by the CLASS developers [13] while the Personal Application Factor was identified by Douglas et al. through factor analysis [19]. In the latter, the starred item (11) was found in the original exploratory factor analysis but did not persist through the confirmatory analysis.

CLASS items
3. I think about the physics I experience in everyday life.
6. Knowledge in physics consists of many disconnected topics.
11. I am not satisfied until I understand why something works
the way it does.
14. I study physics to learn knowledge that will be useful in my life
outside of school.
20. I do not spend more than five minutes stuck on a physics
problem before giving up or seeking help from someone else.
25. I enjoy solving physics problems.
28. Learning physics changes my ideas about how the world works.
30. Reasoning skills used to understand physics can be helpful to
me in my everyday life.
32. Spending a lot of time understanding where formulas come
from is a waste of time.
37. To understand physics, I sometimes think about my personal
experiences and relate them to the topic being analyzed.
40. If I get stuck on a physics problem, there is no chance I'll figure
it out on my own.
42. When studying physics, I relate the important information to
what I already know rather than just memorizing it the way it is
presented.

classified as either (a) to meet a requirement (sample response: "I am taking physics for medical school mostly"), (b) to learn physics (sample response: "I am taking Physics $4 \mathrm{~L}$ because I find physics interesting and the biomedical application of this course is also very appealing. The portion of this course I think I am going to find most interesting is the optics portion, however, electricity and magnetism also seem interesting"), or (c) a combination of both (e.g., "I am taking physics as a premed requirement; however, I am taking [Physics] 4L because I love biology and want to see the application and applicability of physics to biology"); see Table II for numbers in each category.

\section{Interest in physics, and general attitudes towards and beliefs about physics}

In this study, we examine changes both in students' overall CLASS [13] responses and in students' interest in physics, measured using a subset of the CLASS items (discussed in more detail below). Study participants completed the CLASS at the beginning and end of each semester, so all students included in the analysis completed the CLASS four times.

\section{Interest metrics}

We measured student interest in physics using three distinct sets of CLASS items (Table III), and compared the results obtained with each of these measures. We report the standard analysis of the CLASS Personal Interest category to facilitate direct comparison with the existing literature. We also used the Personal Application Factor obtained by Douglas et al. [19] using a factor analysis of CLASS data from nearly 4000 students in introductory calculus-based physics for scientists and engineers. (Because of the relatively small size of our population, factor analysis of our data did not give very robust results, but if we require three factors and accept communalities of 0.4-0.6, one of our factors matches the Personal Application Factor.)

Early in our study, we developed a theoretically derived metric by selecting the twelve items that map onto behavioral indicators of interest as defined through the developmental model, namely, that students' engagement with physics was frequent, deep, voluntary, and independent [44]. This theoretically-based interest metric included the six items assigned to the Personal Interest category designed by the CLASS authors [13] as well as additional items reflecting other dimensions of interest.

The students' scores on all three metrics were correlated $(p<0.001)$, and each of the analyses reported here gave the same qualitative trends and very similar quantitative results regardless of which of these three metrics was used to quantify interest. This agreement of all three metrics in spite of some conceptual differences may reflect that college students are further along in the development of interest than the $\mathrm{K}-12$ population typically studied in motivation research. Nevertheless, the research base for the developmental conception of interest indicates that at any stage, interest may be supported to develop. 
In Sec. V, we report both the results from the CLASS Personal Interest Category (analyzed using the standard \% favorable- $\%$ unfavorable approach) and the Personal Application Factor (analyzed using scores on the 5-point scale after reversing all statements with which an expert would disagree). For multivariate analysis, and for categorizing students as having low, medium, or high initial interest in physics, we use the Personal Application Factor.

\section{Low, medium, and high initial interest in physics}

We divided students into three groups (low, medium, or high) based on their interest in physics at the start of the IPLS class ("initial interest in physics"), as measured by the Personal Application Factor on the 5-point scale. (We confirmed that no significant differences were obtained by using the \% favorable- $\%$ unfavorable analysis of the CLASS to assign students to groups; we used the score on the 5-point scale for greater simplicity in determining $z$ scores.)

We defined the low and high initial interest groups as those students whose scores are 1 standard deviation or more from the mean; the low initial interest group includes 16 out of our sample of 83 students (19\%), and the high initial interest group includes 14 students $(17 \%){ }^{3}$ The medium interest group included all 53 students in between.

\section{E. Interest in life science examples}

On the end-of-semester survey, we asked students to report on whether their interest had been triggered (we used the term "sparked" on the survey ${ }^{4}$ ) by the eight most prominent life science examples used in the IPLS course (boldface in Table I). The following question was followed by the list of examples and a grid on which students rated each example:

\footnotetext{
${ }^{3}$ The following process established the assignments to interest groupings. In our initial analysis of data from Year One, we inspected the distribution of scores for natural breaks at which to divide students into low, medium, and high initial interest; these breaks fell close to $\pm 1 \sigma$ from the mean, although not exactly, and using these breaks placed roughly the bottom and top quartiles of students into the low and high initial interest groups. As the study incorporated more students, the distribution became more continuous, so we defined the low and high initial interest categories as those students whose scores were one standard deviation or more away from the mean, in order to have a simple definition. The same qualitative conclusions, with only minor quantitative differences, are obtained if the low and high groups are enlarged slightly to include the top and bottom quartiles.

${ }^{4}$ Although the accepted term in the motivation literature is "triggered" interest, given the negative connotations of "trigger warnings" on college campuses at the time of the study, it was decided to use "sparked" instead of "triggered" on the survey. "Sparked" is commonly used in the life sciences to refer to triggered interest.
}

"Which of the following examples sparked your interest, and how much? Please rate them from 1 (did not spark interest at all) to 5 (greatly sparked interest)."

To measure each student's interest in the life science examples, we calculated the mean of the eight rankings.

In addition to rating the examples, students were asked to explain why they thought the life science examples engaged their interest. ("Please say a little bit about how the use of life science examples did or did not spark your interest.") Although providing open-ended responses on the survey was optional, $79 \%$ of students gave answers, and of those, $76 \%$ were substantive and provided enough detail to give insight into their thinking. (Those $76 \%$ consisted of $61 \%$ who gave detailed positive responses and $15 \%$ who gave negative or neutral responses; the remaining $24 \%$ gave positive responses that were too vague to interpret.) The detailed positive responses were coded; the categories of responses identified in the coding process $[45,46]$ were as follows:

- Showed interdisciplinary explanatory coherence (i.e., that biology and physics could be used to understand the same thing);

- Revealed the underlying physical mechanism for a previously known phenomenon;

- Showed that physics could explain the "real world";

- Made physics more accessible;

- Showed the future relevance of physics to their planned career.

\section{F. Student course performance}

To characterize student performance in the course, we used composite exam scores (the sum of the scores on both midterms and the final exam). Z-scores were used to create an aggregate distribution of exam scores across the three cohorts of study participants. Exam problems included both qualitative and quantitative problem solving; all exam problems were free response. Problems were graded by the instructor on a rubric established for each problem that accounted for both conceptual understanding and success in quantitative problem solving. Two sample exams are provided in the Supplemental Material [43].

\section{RESULTS AND DISCUSSION}

\section{A. Low, medium, and high initial interest in physics}

Table IV shows the score ranges corresponding to low, medium, and high initial interest in physics, the number of students in each group, and the gender, math preparation, and goals representation in each group. The groups differ by gender distribution and goals. Female students are overrepresented among the low initial interest students and underrepresented among those with high initial interest; this is consistent with historic gender differences on 
TABLE IV. Score ranges and number of individuals corresponding to initial physics interest groups, and gender, math preparation, and goals of each group. Each student's interest score is the mean of his or her pre-IPLS responses to the six items in the Personal Application Factor, on a scale of 1 to 5 $(1=$ strongly disagree, $5=$ strongly agree $)$.

\begin{tabular}{lccc}
\hline \hline $\begin{array}{l}\text { Characteristics of } \\
\text { initial interest groups }\end{array}$ & $\begin{array}{c}\text { Low } \\
(N=16)\end{array}$ & $\begin{array}{c}\text { Medium } \\
(N=53)\end{array}$ & $\begin{array}{c}\text { High } \\
(N=14)\end{array}$ \\
\hline Score ranges & $\begin{array}{c}2.17 \text { to } 3.00 \\
\text { Z-score ranges }\end{array}$ & $\begin{array}{c}3.17 \text { to } 4.00 \\
\text { - } .57 \text { to } \\
-1.0 .780\end{array}$ & 0.714 \\
& -1.08 & 1.01 to 2.51 \\
Gender & & & \\
Male $(N=38,45 \%)$ & $5(31.3 \%)$ & $23(43.4 \%)$ & $10(71.4 \%)$ \\
Female $(N=45,54 \%)$ & $11(68.7 \%)$ & $30(56.6 \%)$ & $4(28.6 \%)$ \\
Math preparation & & & \\
Minimal $(N=24)$ & $4(25.0 \%)$ & $18(34.0 \%)$ & $2(14.3 \%)$ \\
Medium $(N=25)$ & $6(37.5 \%)$ & $15(28.3 \%)$ & $4(28.6 \%)$ \\
High $(N=30)$ & $4(25.0 \%)$ & $18(34.0 \%)$ & $8(57.1 \%)$ \\
Unreported $(N=4)$ & $2(12.5 \%)$ & $2(3.8 \%)$ & $0(0.0 \%)$ \\
Goals & & & \\
Requirement $(N=11)$ & $3(18.8 \%)$ & $7(13.2 \%)$ & $1(7.1 \%)$ \\
Both $(N=38)$ & $8(50.0 \%)$ & $24(45.3 \%)$ & $6(42.9 \%)$ \\
Learning $(N=27)$ & $3(18.8 \%)$ & $17(32.1 \%)$ & $7(50.0 \%)$ \\
Unreported $(N=7)$ & $2(12.5 \%)$ & $5(9.4 \%)$ & $0(0.0 \%)$ \\
\hline \hline
\end{tabular}

CLASS responses [13]. Students with high initial interest were more likely to report learning goals, rather than requirement or combined goals, as measured with an independent $t$ test $(p<0.001)$.

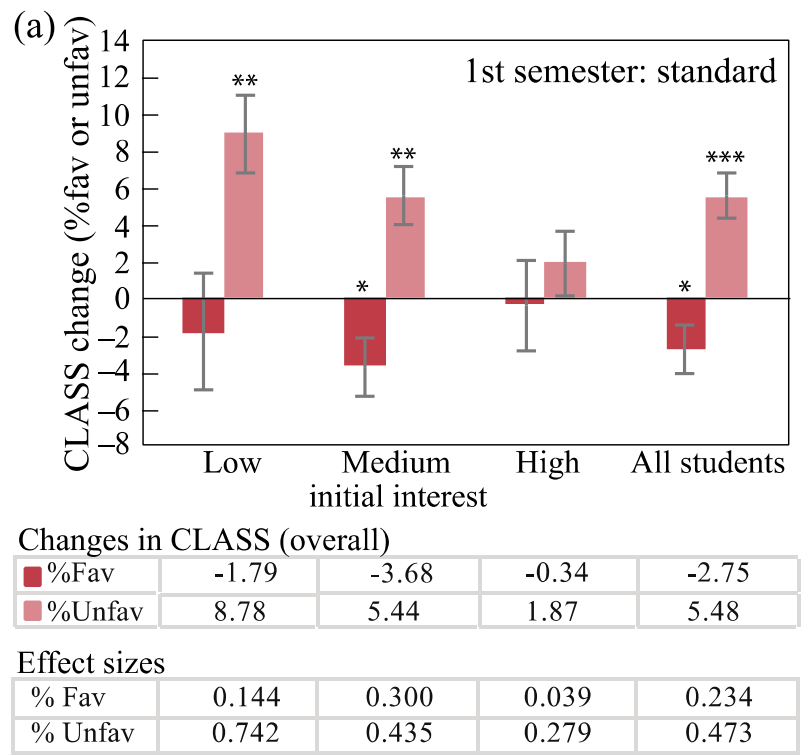

We found no significant difference among the groups in their prior math coursework. We found a marginally significant difference among the groups' physics preparation, as measured by the presemester Brief Electricity and Magnetism Assessment (BEMA) [47]. However, as these scores fall in the range of random guessing, it is unclear whether this difference is meaningful.

\section{B. Improvement of both overall attitudes towards learning physics and interest in physics}

Prior work indicates that after a semester of introductory physics (not necessarily for life science students) students' CLASS responses become less expertlike unless attention is specifically paid to cultivating expertlike attitudes and beliefs about learning physics. This occurs even in courses taught with reformed pedagogies that lead to substantial learning gains $[13,20,48]$. In courses in which attitudes declined in the first semester, in two studies attitudes are observed to decline further during the second semester $[21,22]$, while in one study attitudes are observed to remain steady during the second semester [13]. None of this previous work has broken down the data into different levels of initial interest.

We find that life science students' attitudes toward and interest in physics declined during the standard first semester, but then held steady or slightly improved during the IPLS second semester. Most notably, the students with the lowest initial interest in physics made the greatest gains [49]. All three metrics for interest (presented in Table III) lead to the same conclusion. Figure 1 shows changes in the

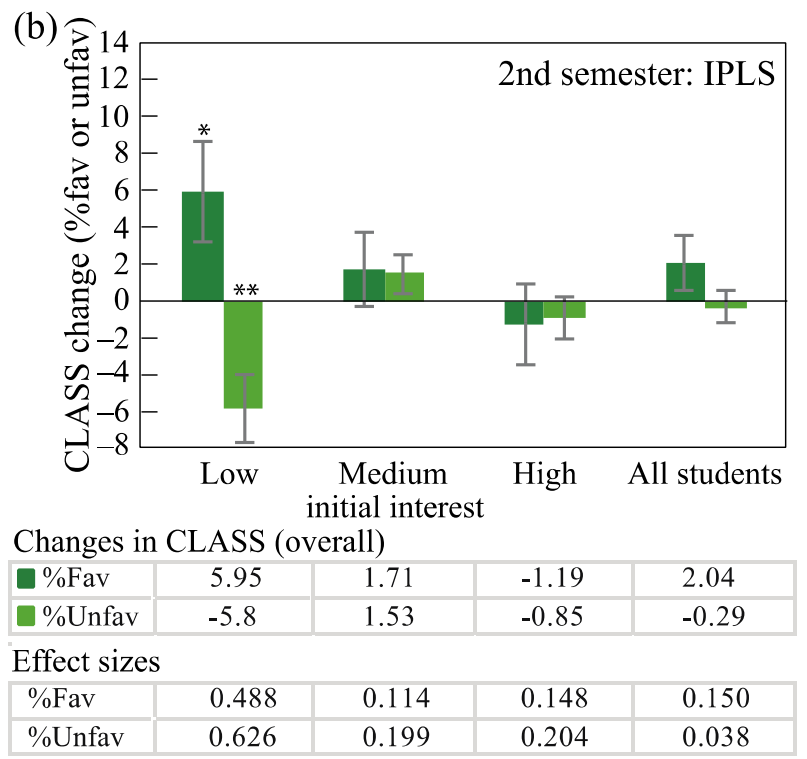

FIG. 1. Changes in overall CLASS responses (post-pre) for all IPLS students with matched data over the three years of the study $(N=83)$, obtained from (a) first semester standard course and (b) second semester IPLS course. Results are shown both for the entire population and for low, medium, or high interest groups (Table IV). Percent favorable and \% unfavorable scoring is done as described in Ref. [13]. The asterisks indicate statistical significance $(* p<0.05, * * p<0.01, * * * p<0.001$ ); error bars show the standard error of the mean. Effect sizes were calculated using Cohen's $d$. The corresponding data are provided in Table S1 [43]. 
CLASS overall for all IPLS students and students in the low, medium, and high interest groups, for the standard first semester and for the IPLS semester.

Figure 2 shows changes in student interest in physics, measured both using the CLASS Personal Interest category (\% favorable or unfavorable), and using the Personal Application Factor (5-point scale). Effect sizes are provided in the minitables directly below the figures. We see that for the first semester, both low and medium initial interest groups show declines in Personal Interest, and the medium group declines in Personal Application. In the IPLS semester, the low initial interest students make gains in both measures, while the medium group holds steady, and the high group declines.

The overall CLASS results (Fig. 1) suggest that the IPLS course also supports the development of students' attitudes toward learning physics more effectively than a standard course. Moreover, the gains in interest made by the low initial interest students suggest that the IPLS course may be particularly effective for supporting them. As we are comparing first and second semester results, to conclusively attribute the improvement in attitudes to the IPLS course will require offering an IPLS first semester course. ${ }^{5}$

Although it is some cause for concern that the students with high initial interest in physics showed a decrease in interest during the IPLS course, examining the pre- and postinterest scores of these students (Table S1, Supplemental Material [43]) revealed that they expressed an extremely high level of interest at the beginning of the IPLS semester (Personal Application Factor mean response of $93 \%$ favorable or 4.48 on the 5-point scale), about 1 standard deviation $\sigma$ higher than they did at the start of the standard semester (preresponse $71 \%$ favorable, or 3.80). As this group showed a decline during the standard first semester (postresponse 63\% favorable, or 3.64), the increase from the end of the first semester to the beginning of the IPLS semester was even more than $1 \sigma$. At the end of the IPLS semester, their interest score (83\% favorable, or 4.12) was still higher ( $p<0.05$ with independent $t$ tests) than it had been at any time in the standard first semester. These analyses suggest that the decline may reflect a ceiling effect in measuring these students' interest, given that these students finished the course with increased interest compared to both their starting and ending responses from the first semester. It is also possible that the students' high interest pre-IPLS reflected anticipation with high expectations.

If meaningful, a possible reason for the observed decline is that these students may have found the course to be insufficiently challenging, and/or felt that the life science applications were not addressed as rigorously as they would have liked. Research indicates that interest can

\footnotetext{
${ }^{5}$ The first semester IPLS course was offered at Swarthmore for the first time in Fall 2015, and will next be offered in Fall 2017 (it is offered only every other year due to staffing limitations).
}
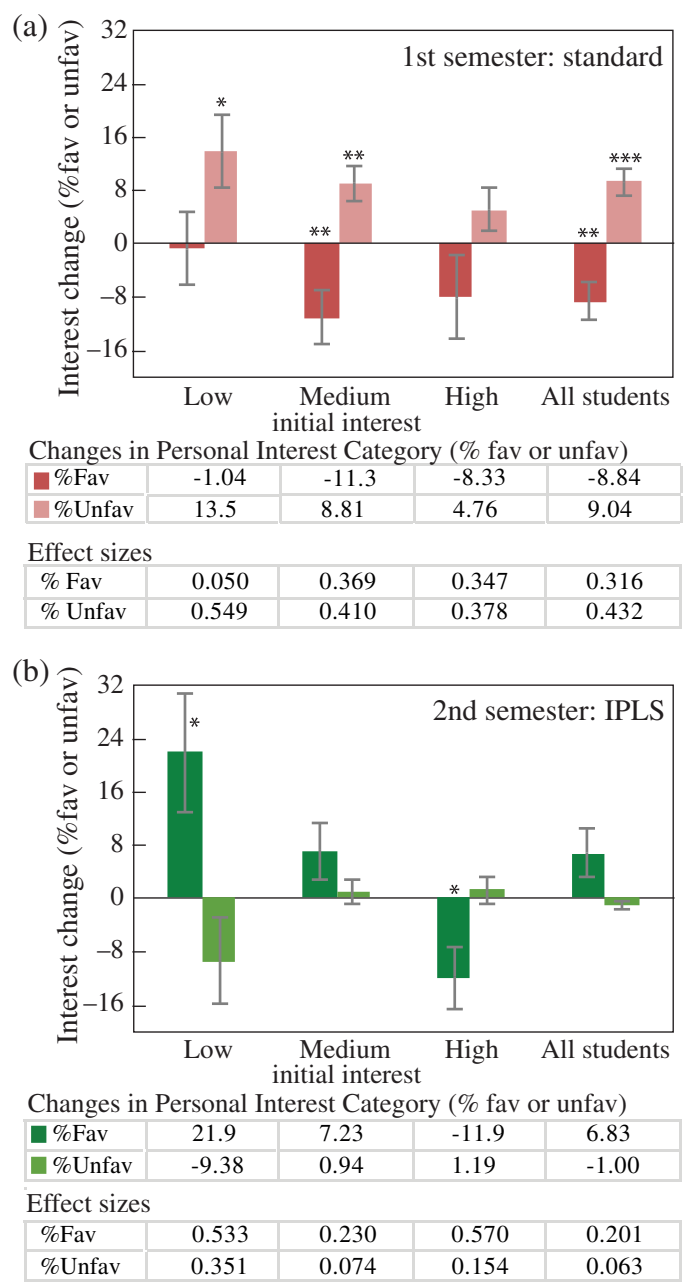

(c)

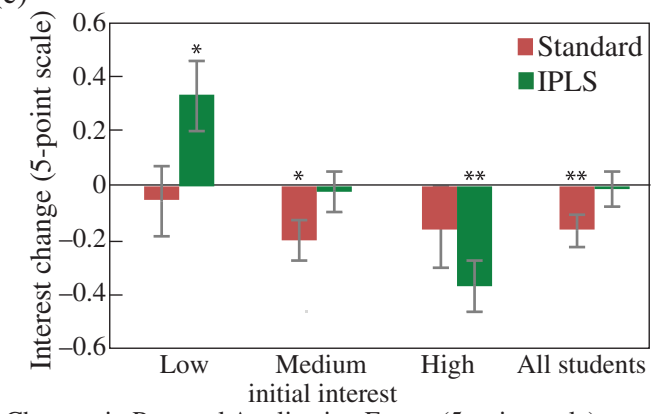

\begin{tabular}{|c|c|c|c|c|}
\hline Standard & -0.052 & -0.195 & -0.157 & -0.161 \\
\hline$\square$ IPLS & 0.333 & -0.020 & -0.364 & -0.010 \\
\hline \multicolumn{5}{|c|}{ Effect sizes } \\
\hline Standard & 0.103 & 0.338 & 0.277 & 0.287 \\
\hline IPLS & 0.555 & 0.035 & 0.734 & 0.017 \\
\hline
\end{tabular}

FIG. 2. Changes in student interest in physics (post-pre), measured using the CLASS Personal Interest category with \% favorable and \% unfavorable (a) for the standard first semester and (b) for the IPLS second semester, and (c) using the Personal Application Factor calculated on the 5-point scale. Results are shown both for the entire population and for low, medium, or high interest groups (Table IV). Asterisks indicate statistical significance $(* p<0.05, * * p<0.01, * * * p<0.001)$; error bars show the standard error of the mean. Effect sizes were calculated using Cohen's $d$. The corresponding data are provided in Table S1 [43]. 
decline if the content with which students are working is insufficiently challenging [12].

\section{Analysis by gender}

Previous work has highlighted that female students typically give less expert responses than male students on the original CLASS Personal Interest category $[13,18]$. In this study, we have examined whether average changes from the start to the finish of the semester differed for male and female students, both in the CLASS overall and in two different interest metrics (CLASS Personal Interest category and Personal Application Factor). We find that male and female students largely exhibit the same changes in interest as the class as a whole, namely, decreasing in the standard course and holding steady in the IPLS course (data presented in Table S2 in the Supplemental Material [43]). For the CLASS Personal Interest category, both males and females show modest improvements in the IPLS semester and declines in the standard semester, although the women show more pronounced declines in the standard semester. The primary observed difference is in the Personal Application Factor during the standard semester; males show no significant change, but females show a significant decline $(p<0.05)$. Both males and females show no significant change in the Personal Application Factor during the IPLS semester.

These results are consistent with the work of Hoffman and Häussler, which showed that female students learned comparably to male students when physics concepts were taught using life science contexts which were of comparable interest to both male and female students [9]. Similarly, we conjecture that male and female students have comparable interest in the life sciences, and thus will experience comparable effects in the reformed IPLS course.

We do see lower Personal Interest and Personal Application Factor scores from female students, both presemester and postsemester, and for both standard and IPLS semesters. This is consistent with the low initial interest group including a disproportionate number of female students (Table IV).

Our sample size is not big enough to allow a meaningful analysis of the low, medium, and high groups by gender. However, we do observe that the low initial interest students, who are disproportionately female, make the greatest gains in attitudes and interest in the IPLS semester.

\section{Contributions of example interest and prior physics interest to course performance}

When learners develop an interest in a discipline such as physic they are motivated to learn, which has a positive influence on course performance [12]. In the present study, we examined whether the integral role of the life science examples influenced students' course performance. Analysis of variance indicated that example interest was a significant predictor of exam score, whereas initial interest in physics was not, as shown in Fig. 3. (Exams
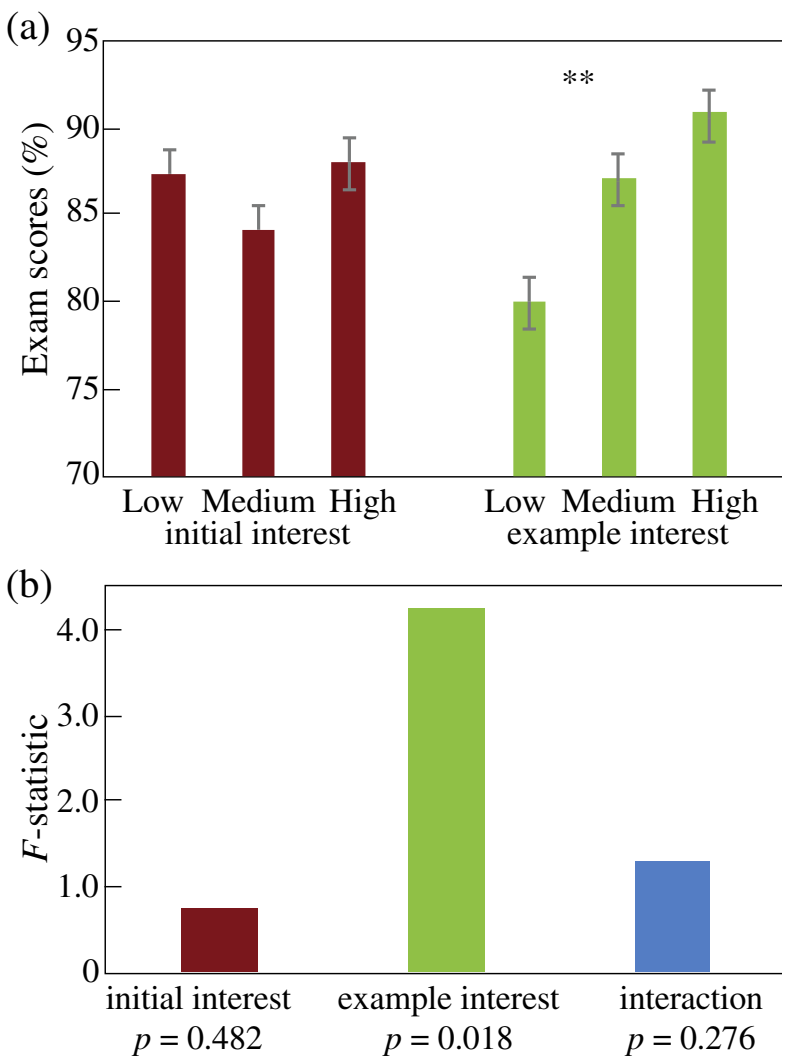

FIG. 3. (a) Average composite exam scores for initial interest groups (Personal Application Factor) and example interest groups. (** indicates that low, medium, and high example interest groups differ with statistical significance, $p<0.01$ ) (b) $F$ statistic for ANOVA conducted on composite examination scores, testing the contributions of initial interest in physics and example interest (low, medium, or high; measured as described in Secs. IV. D. 2 and IV. E) to the variance in scores.

included both problems that connected to the life science applications and problems that had no life science application. Representative exams are provided in the Supplemental Material [43].)

This analysis suggests that students whose interest was supported by the life science examples benefited from the IPLS course being designed to develop students' knowledge of such examples and ability to apply physics to the life sciences themselves throughout the course. As this analysis demonstrates only correlation, not causation, further work is needed to determine whether this is the case.

This finding is also consistent with students' end-ofcourse survey responses that the presence of the life science examples made the course more interesting to them. In Years Two and Three of the study, a Likert scale rating statement was added to the survey: "Compared to teaching the same physics without the biological, biochemical, and medical examples, by including these examples, the course was ..." with the five choices ranging from "much less interesting to me" to "much more interesting to me." In both years, about $90 \%$ of the entire class chose either 
"much more interesting" or "somewhat more interesting" (Year Two: 60\% "much more" and 31\% "somewhat"; Year Three: $67 \%$ "much more," 23\% "somewhat"), with the others choosing "equally interesting," except for one student in Year Two choosing "somewhat less interesting."

\section{E. Understanding how students respond to particular examples}

Clearly an important next step in the study of the IPLS course is understanding what makes particular life science examples interesting to students, both to inform instructors and developers of such courses and to connect to the existing literature on interest $[45,46]$. We analyzed the rates at which low, medium, and high initial interest students gave each category of open-ended response (Table V). Among the detailed positive responses, the most frequent category for all initial physics interest levels was explanatory coherence, given by all groups at comparable rates ( $24 \%$ from L and H, 26\% from M). Examples of such responses include the following:

"The membrane potential and nerve signaling was neat as we were covering them in neurobiology from a different perspective at the same time."

"I thought optics and myopia was particularly interesting. I also enjoyed the examples with gel electrophoresis (SDS-PAGE), especially since they coincided with what we were learning in biochemistry and provided an alternate perspective on the topic."

For the medium and high initial interest groups, the rate of responses in the real-world connections category was also comparable ( $24 \%$ for both); for the low initial interest group, although the real-world connections category was the second most frequent, it occurred at a significantly

TABLE V. Rates of categories of open-ended responses by initial physics interest categories, from matched data set. (Five students who are part of the matched data set did not provide open-ended responses and thus are not represented) Full data set of open-ended responses includes 123 total responses and shows equivalent results.

\begin{tabular}{lccc}
\hline \hline & \multicolumn{3}{c}{ Answer rate by physics initial interest } \\
\cline { 2 - 4 } & Low & Medium & High \\
Category & $(N=17)$ & $(N=34)$ & $(N=17)$ \\
\hline Explanatory coherence & $24 \%$ & $26 \%$ & $24 \%$ \\
Underlying mechanism & $0 \%$ & $12 \%$ & $24 \%$ \\
Real world application & $12 \%$ & $24 \%$ & $24 \%$ \\
Ease or accessibility & $0 \%$ & $0 \%$ & $18 \%$ \\
Future relevance & $6 \%$ & $3 \%$ & $0 \%$ \\
None & $12 \%$ & $9 \%$ & $6 \%$ \\
Neutral or negative & $35 \%$ & $3 \%$ & $6 \%$ \\
Vague positive & $18 \%$ & $38 \%$ & $24 \%$ \\
\hline \hline
\end{tabular}

lower rate (12\%), suggesting that for students with low initial physics interest, the biological connections were more significant than other types of familiar examples. (It is worth noting that for the second semester topics of optics, electricity, and magnetism, most of the nonbiological realworld connections are technological and might not be as salient to life science students as real-world connections to mechanics; repeating this kind of analysis with a first semester IPLS course might reveal a different pattern for the real-world examples.)

Students with high initial physics interest were significantly more likely than the others to give responses in the underlying physical mechanism category (24\%); for the high initial interest group, underlying mechanism and explanatory coherence responses occurred at the same frequency. For example, a student with high initial interest in physics explained,

"Knowing about the biological phenomena involved helped me better appreciate having a different approach than the one I had already learned. For example, I don't think screening in salt solution would have been as interesting to me if I hadn't already known a bit about DNA, proteins, and cells. I loved the [homework] problem asking us to calculate the electric forces between DNA molecules in pure water as screening was being introduced and we found that the electric forces should be too great for so much DNA to be packed together in the nucleus. This was a turning point for me, as I had never considered this before, but now that I had, it seemed to be a very significant problem. If I hadn't known anything about DNA, this would not have been as worthwhile of a problem."

Another student with high initial interest noted,

"For example, when we learned about immersion oil in microscopy, I had already had experience using this technique, and I knew that it was to increase the amount of light that went into the microscope, but I didn't really understand how oil helped until we talked about the example in class. In general, I found that I was familiar with the examples used because of my previous courses in biology and chemistry, but I had a weak or incomplete understanding of the physics behind the examples until this class."

In general, the open-ended responses of the students are consistent with the findings of Hoffmann and Häussler [9]: they suggest that the use of life science examples best supports the development of student interest when those examples extend their present understanding. These data suggest further that students are best prepared to have their interest engaged when they can make a sufficient connection to the content being learned. Furthermore, it appears that for students with less developed initial interest 
in physics, such connections matter most by building a broad level of coherence, whereas for those with more developed initial interest, the details of the physical mechanism are also important.

Detailed analysis of the open-ended responses, reported elsewhere $[45,46]$, holds promise for better understanding both the mechanism by which the life science examples engage student interest and how to effectively engage students in the IPLS course who have differing degrees of initial interest in physics.

\section{CONCLUSIONS AND IMPLICATIONS FOR INSTRUCTION}

We have described findings from a first study of an IPLS course, in which introductory physics was taught embedded in authentic life science examples, and students' skills in applying physics to the life sciences were explicitly developed. Our findings indicate that in this context, students' interest in the examples predicts their exam score more accurately than their pre-IPLS interest in physics, and that students' interest in and attitudes towards physics increase over the IPLS semester. We interpret our findings as suggesting that using life science examples in this manner as the context for learning physics can support physics performance, support more expert-like attitudes as measured by the CLASS, and trigger the development of student interest, especially for students who enter the IPLS course with low initial interest in physics. Our findings are consistent with prior work in which content of interest to participants is used as the context of instruction $[9,31,32]$.

Our finding that students with high initial interest in physics show modest declines in their physics interest over the semester sounds a cautionary note, although it may solely reflect a ceiling effect or student anticipation. The interest literature suggests that for interest to continue to develop and deepen, students need to be challenged [12]. Further study to better understand the interaction between students' level of interest in physics and the use of life science examples is needed. It is possible that a more sophisticated treatment of the life science examples would have better engaged the interest of the students with high initial physics interest; the open-ended responses suggest that simply including a life science example may not support them as effectively as would an example that pushes them to deepen their understanding of both the physics and the life science content of the example. Future curriculum development might include differentiated assignments designed to allow students who have greater initial interest in physics and/or more facility with mathematics to solve more challenging biological problems, or to work on project-type assignments that allow them to pose and investigate their own questions.

\section{ACKNOWLEDGMENTS}

We thank Benjamin Geller for sharing his coding of the open responses; Suzanne Hidi for feedback on P. W. 's honors thesis at Swarthmore College, which provided a starting point for some of the analysis reported here; and Ann Ruether for assistance with data management. C. H. C. also acknowledges helpful conversations about IPLS course design and assessment with Benjamin Geller, Kristi Hall, Vashti Sawtelle, and the University of Maryland NEXUS team; and about the CLASS with Wendy Adams, Adrian Madsen, Sarah McKagan, and Kathy Perkins. This work was supported by a grant to Swarthmore College from the Howard Hughes Medical Institute through the Precollege and Undergraduate Science Education Program, and by NSF Grant No. DUE-1122941.
[1] Committee on Undergraduate Biology Education, National Research Council, BIO 2010: Transforming Undergraduate Education for Future Research Biologists (National Academies Press, Washington, DC, 2003).

[2] American Association for the Advancement of Science, Vision and change in undergraduate biology education: A call to action (AAAS Press, Washington, DC, 2011).

[3] Howard Hughes Medical Institute-American Association of Medical Colleges Committee, Scientific Foundations for Future Physicians (American Association of Medical Colleges, Washington, DC, 2009).

[4] See, for example, the programs for the sessions Reforming the Introductory Physics Course for Life Science Students held at the AAPT national meetings since Summer 2009, www.aapt.org/AbstractSearch, the CBE-LSE issue mentioned in Ref., and the IPLS Wiki, www.phys.gwu.edu/ iplswiki, with documents from the NSF-funded 2009 workshop.

[5] D. C. Meredith and E. F. Redish, Reinventing physics for life-sciences majors, Phys. Today 66, No. 7, 38 (2013).

[6] See for example M. Sabella and M. Lang, Research and education at the crossroads of physics and biology, Am. J. Phys. 82, 365 (2014), and other articles in this theme issue.

[7] J. E. Watkins, K. L. Hall, J. E. Coffey, T. J. Cooke, and E. F. Redish, Disciplinary authenticity: Enriching the reform of introductory physics courses for life science students, Phys. Rev. ST Phys. Educ. Res. 8, 010112 (2012).

[8] C. S. Hulleman and J. M. Harackiewicz, Promoting interest and performance in high school science classes, Science 326, 1410 (2009). 
[9] L. Hoffmann and P. Häussler, An intervention project promoting girls', and boys' interest in physics, in Interest and Learning, edited by L. Hoffmann, A. Krapp, K. A. Renninger, and J. Baumert (Institut für die Pädagogik der Naturwissenschaften, Kiel, Germany, 1998), pp. 301-316.

[10] S. Hidi and K. A. Renninger, The four-phase model of interest development, Educ. Psychol. 41, 111 (2006).

[11] K. A. Renninger and S. Hidi, Revisiting the conceptualization, measurement, and generation of interest, Educ. Psychol. 46, 168 (2011).

[12] K. A. Renninger and S. E. Hidi, The Power of Interest for Motivation and Engagement (Routledge, New York, 2016).

[13] W. K. Adams, K. K. Perkins, N. S. Podolefsky, M. Dubson, N. D. Finkelstein, and C. E. Wieman, New instrument for measuring student beliefs about physics and learning physics: The Colorado Learning Attitudes about Science Survey, Phys. Rev. ST Phys. Educ. Res. 2, 010101 (2006).

[14] E. Seymour and N. Hewitt, Talking about Leaving (Westview Press, Boulder, CO, 1997).

[15] E. Redish, J. M. Saul, and R. N. Steinberg, Student expectations in introductory physics, Am. J. Phys. 66, 212 (1998).

[16] D. Hammer, Student resources for learning introductory physics, Am. J. Phys. 68, S52 (2000).

[17] J. D. Bransford, A. L. Brown, and R. R. Cocking, How People Learn (National Academy Press, Washington, DC, 2002).

[18] C. E. Wieman and W. Adams, On the Proper Use of Statistical Analyses; a Comment on "Evaluation of Colorado Learning Attitudes about Science Survey", arXiv:1501.03257.

[19] K. A. Douglas, M. S. Yale, D. E. Bennett, M. P. Haugen, and L. A. Bryan, Evaluation of colorado learning attitudes about science survey, Phys. Rev. ST Phys. Educ. Res. 10, 020128 (2014).

[20] A. Madsen, S. B. McKagan, and E. Sayre, How physics instruction impacts students' beliefs about learning physics: A meta-analysis of 24 studies, Phys. Rev. ST Phys. Educ. Res. 11, 010115 (2015).

[21] L. Kost-Smith, S. Pollock, and N. Finkelstein, Gender disparities in second-semester college physics: the incremental effects of a 'smog of bias', Phys. Rev. ST Phys. Educ. Res. 6, 020112 (2010).

[22] C. H. Crouch and K. Heller, Introductory physics in biological context: An approach to improve introductory physics for life science students, Am. J. Phys. 82, 378 (2014).

[23] K. K. Perkins, W. K. Adams, N. D. Finkelstein, S. J. Pollock, and C. E. Wieman, Correlating student attitudes with student learning using the Colorado Learning Attitudes about Science Survey, AIP Conf. Proc. 790, 61 (2005).

[24] G. Kortemeyer, Correlations between student discussion behavior, attitudes, and learning, Phys. Rev. ST Phys. Educ. Res. 3, 010101 (2007).

[25] K. C. Berridge, T.E. Robinson, and J. W. Aldridge, Dissecting components of reward: 'liking,' 'wanting,' and learning, Curr. Opin. Pharmacology 9, 65 (2009).

[26] K. A. Renninger, C. C. Kensey, S. J. Stevens, and D. L. Lehman, Perceptions of science and their role in the development of interest, in Interest in mathematics and science learning, edited by K. A. Renninger, M. Nieswandt, and S. Hidi (AERA, Washington, DC, 2015), 93-110.

[27] M. Ainley and L. Patrick, Measuring self-regulated learning processes through tracking patterns of student interaction with achievement activities, Educ. Psychol. Rev. 18, 267 (2006).

[28] D. Palmer, Research report: Situational interest and the attitudes towards science of primary teacher education students, Int. J. Sci. Educ. 26, 895 (2004).

[29] D. H. Palmer, Student interest generated during an inquiry skills lesson, J. Res. Sci. Teach. 46, 147 (2009).

[30] C. Sansone, What's interest got to do with it? Potential trade-offs in the self-regulation of motivation, in Psychology of Self-Regulation: Cognitive, Affective, and Motivational Processes, edited by J. P. Forgas, R. Baumiester, and D. Tice (Psychology Press, New York, 2009), pp. 35-51.

[31] K. A. Renninger, L. Ewen, and A. K. Lasher, Individual interest as context in expository text and mathematical word problems, Learn. Instr. 12, 467 (2002).

[32] C. Walkington, A. Petrosino, and M. Sherman, Supporting algebraic reasoning through personalized story scenarios: How situational understanding mediates performance, Math. Thinking Learn. 15, 89 (2013).

[33] M. Mitchell, Situational interest: Its multifaceted structure in the secondary school mathematics classroom, J. Educ. Psychol. 85, 424 (1993).

[34] C. Randler and F.X. Bogner, Pupils' interest before, during, and after a curriculum dealing with ecological topics and its relationship with achievement, Educ. Res. Eval. 13, 463 (2007).

[35] K. A. Renninger, C. C. Kensey, S. J. Stevens, and D. L. Lehman, Perceptions of Science and their Role in the Development of Interest, in Interest in mathematics and science learning, edited by K. A. Renninger, M. Nieswandt, and S. Hidi (AERA, Washington, DC, 2015), pp. 93-110.

[36] R. A. Engle, Framing interactions to foster generative learning: A situative explanation of transfer in a community of learners classroom, J. Learn. Sci. 15, 451 (2006).

[37] R. A. Engle, P. D. Nguyen, and A. Mendelson, The influence of framing on transfer: Initial evidence from a tutoring experiment, Instr. Sci. 39, 603 (2011).

[38] Based on L. McDermott and P. Shaffer (the University of Washington Physics Education Group), Tutorials in Introductory Physics, 2nd ed. (Pearson, Upper Saddle river, NJ, 2013), pp. 185-207.

[39] C. H. Crouch, J. Watkins, A. P. Fagen, and E. Mazur, Peer Instruction: Engaging students one-on-one, all at once, in Research-Based Reforms in University Physics, edited by E. F. Redish (American Association of Physics Teachers, College Park, MD, 2007), pp. 1-55, http://www.compadre .org/PER/items/detail.cfm?ID=4990.

[40] P. Heller, R. Keith, and S. Anderson, Teaching problem solving through cooperative grouping. Part 1: Group versus individual problem solving, Am. J. Phys. 60, 627 (1992).

[41] P. Heller and M. Hollabaugh, Teaching problem solving through cooperative grouping. Part 2: Designing problems and structuring groups, Am. J. Phys. 60, 637 (1992).

[42] P. Heller, K. Heller, and T. Foster, Cooperative group problem solving laboratories for introductory classes, AIP Conf. Proc. 399, 913 (1996). 
[43] See Supplemental Material at http://link.aps.org/ supplemental/10.1103/PhysRevPhysEducRes.14.010111 for syllabi with topics and biological contexts, sample exam problems, and Tables S1 and S2.

[44] K. A. Renninger and R. H. Wozniak, Effect of interests on attentional shift, recognition, and recall in young children, Dev. Psychol. 21, 624 (1985).

[45] B. Geller, C. Turpen, K. A. Renninger, P. Wisittanawat, and C. H. Crouch, Unpacking the source of student interest in an IPLS course, abstract available at http://www.compadre .org/per/perc/2015/Detail.cfm?id=6225.

[46] B. Geller, C. Turpen, and C. H. Crouch, Sources of student engagement in introductory physics for life sciences [Phys. Rev. Phys. Ed. Res. (to be published)].
[47] L. Ding, R. Chabay, B. Sherwood, and R. J. Beichner, Evaluating an electricity and magnetism assessment tool: brief electricity and magnetism assessment, Phys. Rev. ST Phys. Educ. Res. 2, 010105 (2006).

[48] Similar MPEX results are reported in G. Kortemeyer, The challenge of teaching introductory physics to premedical students, Phys. Teach. 45, 552 (2007).

[49] Preliminary analysis of a single year of data was previously presented, C. H. Crouch, P. Wisttanawat, and K. A. Renninger, Initial interest, goals, and changes in CLASS scores in introductory physics for life sciences, in Proceedings of the Physics Education Research Conference 2013, Portland, Oregon, edited by P. Engelhardt, A. Churukian, and D. Jones (AIP, New York, 2013). 